\title{
A 5 CURVE THEOREM GENERALIZING THE THEOREM OF CARNOT
}

\author{
TH. MOTZKIN
}

Because of the 2-dimensionality of the plane a relation holds between any 3 quantities determining a point or a line. The relation between the meets of a line with the sides of a fixed triangle is given by the theorem of Menelaos. This is generalized to the meets of an algebraic curve with a triangle by the theorem of Carnot. I give here a further generalization to the meets of an algebraic curve with a triangle whose sides are arbitrary algebraic curves. The formulation of the theorem requires the introduction of the new notion of curve cross ratio; it will be verified by a simple algebraic method allowing us to prove a wide class of allied theorems. The theorem itself is already very extensive; of the numerous special cases, which include many known theorems, I can, in this place, only give a few examples, but I give an outline of the main procedures in obtaining, step by step, special cases and related theorems.

The theorem of Carnot states that the cyclic product of the ratios in which the sides of a polygon are divided by an algebraic curve is 1 : it may be written $I I(A P: B P)=1$, if $A$ is a vertex, $B$ the next vertex, and $P$ a meet of the curve and $A B$. Carnot's theorem, which Poncelet in his epoch-making treatise Propriétés projectives des figures, a great part of which is based upon it, calls "le principe fondamental," expresses, as I shall show elsewhere, a characteristic property of algebraic curves; it may also be extended to a class of limiting cases.

The usual proofs of Carnot's theorem are by reduction to one of two special cases. The polygon is decomposed either into triangles or into quadrangles with two opposite vertices in the infinite, and these cases (the second of which is a theorem of Newton) are proved by writing the free term of a polynomial as product of its roots.

Menelaos' theorem is usually proved by homothetic triangles (the simplest case of Newton's theorem); it may also be obtained by dualizing the theorem of Ceva. The latter is a trivial corollary of the representation of a point $P$ by barycentric coordinates, with respect to a triangle $A B C$ : if $A P$ meets $B C$ at $A^{\prime}$, and so on, we have $A C^{\prime}: B C^{\prime}=A C C^{\prime}: B C C^{\prime}$ (areas) $=A C P: B C P=A C P:-C B P ;$ and, evidently, the cyclic product of the ratios analogous to the last is -1 . Menelaos' theorem, and its exact dual equivalent, may likewise be expressed as identities between areas of triangles, that is, between

Received by the editors November 2, 1944. 
determinants. Now a determinant is only the simplest case of the resultant of 3 quantics, and this leads us to the formulation and proof of the generalized theorem.

We consider Möbius' 5 line theorem. If each ratio in Menelaos' theorem is considered as a cross ratio with one point at the infinite, we obtain a projective theorem on two transversals of a triangle: the cyclic product of the cross ratios on the sides is 1 . Exactly the same statement holds for 5 general algebraic curves (here the 4 curve theorem obtained by choosing the line at infinity as 5th curve is a very special case from which the general theorem cannot be deduced by projective generalization). This statement will make sense as soon as we define the cross ratio determined on a curve (side) by 4 curves ( 2 sides and 2 transversals).

Let the given side (and the quantic vanishing there) be $a$, the adjacent sides $b$ and $c$, the transversals $p$ and $q$. Denoting by $a b p$ the resultant of the 3 quantics, the required cross ratio $a(b p / c q)$ shall be defined as $(a b p: a b q):(a c p: a c q)$. It has certain interesting properties, and its definition is a natural one, as seen by the following consideration. Denote the set of meets of $a$ and $p$ by $a p$, and so on. In case of lines, the cross ratio of the mutual distances of $a b, a c, a p, a q$ equals the cross ratio of the distances of $a b$ and $a c$ from $p$ and $q$. The last cross ratio may be obtained by substituting the values of $a b$ in $p$, and so on. In the general case, substitution of every point $x$ of $a b$ in $p$ and multiplication $\Pi p(x)$ of the results gives $a b p$.

Remarks. 1. In the definition of the curve cross ratio, as throughout the note, we make the tacit assumption that no resultant which is explicitly mentioned is 0 , that is, that the corresponding 3 curves do not have a common point. 2. If $b$ and $c$, and if $p$ and $q$ are of equal degree, the curve cross ratio is not affected by multiplication of the quantics by constants, and thus depends only on the given curves. The simple ratio $r=a b p: a b q=\Pi(p(x): q(x))$, on the other hand, may differ for proportional quantics. For $p$ and $q$ of equal degree, all $x$ with a fixed $r=-\mu: \lambda$ form a curve $\lambda p+\mu q=0$.

The 5 curve theorem follows at once from the definition: $a(b p / c q) \cdot b(c p / a q) \cdot c(a p / b q)= \pm 1$, as all factors cancel out, or in words :

The cyclic product of the cross ratios determined on the sides of a curvilinear triangle by two curvilinear transversals is \pm 1 .

The same relation $\prod a_{k}\left(a_{k-1} p / a_{k+1} q\right)= \pm 1$ holds for polygons.

We now prove the 4 curve theorem. In the preceding identities, already the product of the terms containing $p$ equals \pm 1 , and the 
second transversal is needed only to complete the cross ratios. If $a, b, c$ are given and if a fixed $q$ is chosen, we obtain a relation between the meets of $p$ with $a, b, c$. For example, if the meets $a p$ and $b p$ are given, we obtain a certain value $v$ of $a c p: b c p$; that is, the product $v$ of the ratios of the "distances" of the points $c p$ from $a$ and $b$ (as $-\mu: \lambda$ in Remark 2) remains unaltered if we change the curve $p$, as long as $a p$ and $b p$ remain fixed. Exchanging the rôle of $p$ and $c$, we see that $v$ also retains its value if $c$ is modified without changing $a c$ and $b c$. so we have:

The meets of two curves $c$ and $d$ with two given curves $a$ and $b$ of equal degrees determine the product acd:bcd of the distances of the meets of $c$ and $d$ from $a$ and $b$.

If the degrees $\alpha$ and $\beta$ of $a$ and $b$ are different, the value of $a c d^{\beta}: b c d^{\alpha}$ is determined.

Examples of kindred theorems. Quotients of products of resultants in which all terms cancel (except the sign) can, of course, be formed in various ways. As examples we formulate the 2 following theorems:

I. The cyclic product of the ratios determined on each side of a curvilinear polygon by all non-adjacent sides is 1 .

II. The cyclic product of the ratios of the "segment" determined on each side of a curvilinear polygon by the following two sides to that determined on the same side by the two preceding sides is 1 .

Indeed, let the sides in their order be $a, b, c, \cdots, p, \cdots$. The product in I is composed of ratios $a b p: b c p$ with $p \neq a, b, c$. All factors cancel out (the form $a b c$ is cancelled by $c a b$ ). The factors of the product in II are of the form $c a b: c d e$ and cancel already on alternating sides; but to complete them to cross ratios (for use, for example, in the linear case) they must be written $c a b: c b d \cdot c b d: c d e:(c a q: c q d \cdot c b q: c q e)$, and the additional terms will cancel only if all the sides are considered.

Other notions related to the curve cross ratio which may occur in theorems of the same type are:

(1) The cyclic ratio on a curvilinear polygon, that is, the cyclic product of the ratios determined on each side $s_{k}$ by a corresponding curve $p_{k}$;

(2) The cyclic ratio $a b_{i} c_{j}: a c_{j} b_{j+1}$ on a curve $a$ (this generalization of the cross ratio is a product of cross ratios);

(3) The triple cross ratio $a_{j} b_{k} c_{l}^{(-1)^{i+k+l}}, j, k, l=1,2$, of 6 curves.

We now discuss methods for deriving other theorems and special cases. Starting with the 5-curve theorem (or a related one), we proceed by one or more of the following steps: 
1. Dualization.

2. (a) Affine specialization by a suitable choice of the line at infinity; (b) metrical specialization by a suitable choice of the isotropic points $I, J$.

3. (a) Specialization of the polygon; (b) composition of polygons.

4. (a) Specialization of curves to cubics, conics and lines; (b) specialization to reducible curves.

5. Passing to limiting cases with one or more vanishing resultants.

Example: orientation of common tangents. By the steps $4 \mathrm{a}, 1$ and $2 \mathrm{~b}$, we derive a theorem of Laguerre from the 4 curve theorem. Let $a$ and $b$ in 9 be lines; after dualizing they become points. Choose them as $I$ and $J$. As fixing the tangents to a curve from $I$ and $J$ is equivalent to fixing its foci, and as the product of the ratios of the "distances" of given lines from $I$ and $J$ determines their orientation, ${ }^{1}$ we have:

The orientation of the common tangents of two curves is determined by their foci.

It is the same as the orientation of the lines connecting the foci of one curve with those of the other.

We now pass to a single curve. From the 4 curve theorem with $a$ and $b$ as lines we derive a theorem on $a, b$ and a single curve $c$. Let $p$ be the first polar of $a b$ with regard to $c$; the meets $a p$ may be determined from $a c$ only, and $b p$ from $b c$. As the meets $c p$ are the points of contact on the tangents to $c$ from $a b$, we have:

The meets of a curve $c$ with two given lines $a, b$ determine the ratio product (with regard to $a$ and $b$ ) of the tangents from $a b$.

Conversely, this theorem implies the 4 curve theorem with $a$ and $b$ as lines: take $c$ as a reducible curve $d \cdot e$, and apply the theorem to $c, d, e$ separately, then note that the tangents to $c$ are the same as to $d$ and $e$ plus the lines to $d e$.

Dualizing the preceding theorem, replacing $a$ and $b$ by $I$ and $J$ and remarking that the ratio $c(I): c(J)$ determines the orientation ${ }^{2}$ of $c$, we see that the orientation of a curve is determined by its foci.

UNIVERSITY OF JERUSALEM

\footnotetext{
${ }^{1}$ Sum of angles with a given line.

${ }^{2}$ Sum of angles of the asymptotes with a given line.
} 